\title{
Proper stellar directions and astronomical aberration
}

\author{
Mariateresa Crosta ${ }^{1}$ and Alberto Vecchiato ${ }^{1}$ \\ ${ }^{1}$ Osservatorio Astronomico di Torino-INAF \\ email: crosta@oato.inaf.it, vecchiato@oato.inaf.it
}

\begin{abstract}
The general relativistic definition of astrometric measurement needs an appropriate use of the concept of reference frame, which should then be linked to the conventions of the IAU Resolutions (Soffel et al., 2003), which fix the celestial coordinate system. A consistent definition of the astrometric observables in the context of General Relativity is also essential to find uniquely the stellar coordinates and proper motion, this being the main physical task of the inverse ray tracing problem. Aim of this work is to set the level of reciprocal consistency of two relativistic models, GREM and RAMOD (Gaia, ESA mission), in order to guarantee a physically correct definition of light direction to a star, an essential item for deducing the star coordinates and proper motion within the same level of measurement accuracy.
\end{abstract}

Keywords. space astrometry, relativity, reference systems, catalogs, solar system, gravitation

\section{Introduction}

Modern space mission like Gaia (Turon et al., 2005) requires that any astrometric measurement has to be modelled in a way that light propagation and detection are both conceived in a general relativistic framework. One needs, in fact, to solve the relativistic equations of the null geodesic which describes the trajectory of a photon emitted by a star and detected by an observer with an assigned state of motion. The whole process takes place in a geometrical environment generated by an N-body distribution as could be that of our Solar System.

The astrometric problem consists in the determination, from a prescribed set of observational data (hereafter observables) of the astrometric parameters of a star, namely its coordinates, parallax, and proper motion. Essential to the solution of the inverse ray tracing is the identification, as boundary conditions, of the local observer's line-ofsight defined in a suitable reference frame. However, while in classical (non relativistic) astrometry these quantities are well defined, in General Relativity (GR) they must be interpreted consistently with the relativistic framework of the model. Similarly, the parameters describing the attitude and the center-of-mass motion of the satellite need to be defined consistently with the chosen relativistic model.

At present, two conceptual frameworks are foreseen for Gaia. The first model, named GREM (Gaia Relativistic Model) and described in Klioner, 2003, has been formulated according to a Parametrized Post Newtonian (PPN) scheme accurate to 1 micro-arcsecond. GREM is considered as baseline for the Gaia data reduction. The second model, RAMOD (Relativistic Astrometric MODel), is conceived to solve the inverse ray-tracing problem in a general relativistic framework not constrained a priori by any target accuracy. At present, the RAMOD full solution requires the integration of a set of coupled non linear differential equations which allows to trace back the light trajectory to the initial position of the star and which naturally entangles, all together, the contributions by the aberration and all those due to the curvature of the background geometry. 
Since both models are used for the Gaia data reduction with the purpose to create a catalog of positions and proper motions based on measurements of absolute astrometry, any inconsistency in the relativistic model(s) would invalidate the quality and reliability of the estimates, indeed all the related scientific outputs. This alone is sufficient reason for making a theoretical comparison of the existing approaches for Gaia a necessity.

In this work we present the first step of the theoretical comparison, showing how it is possible to isolate the aberration terms from the global RAMOD construct and recasting them in a GREM-like formula.

\section{The RAMOD frames}

The set-up of any astrometric model implies, primarily, the identification of the gravitational sources and of the background geometry. Then one needs to label the space-time points with a coordinate system. The above steps fix a reference frame with respect to which one describes the light trajectory, the motion of the stars and that of the observer.

The RAMOD framework is based on the weak-field requirements for the background geometry, which in turn has to be specialized to the particular case one wants to model. For a Gaia-like mission, we can assume the Solar System as the only source of gravity, i.e. a physical system gravitationally bound, in the weak field and small velocities regime. With these assumptions the background geometry is given by the following line element $\mathrm{d} s^{2} \equiv g_{\alpha \beta} \mathrm{d} x^{\alpha} \mathrm{d} x^{\beta}=\left(\eta_{\alpha \beta}+h_{\alpha \beta}+\mathcal{O}\left(h^{2}\right)\right) \mathrm{d} x^{\alpha} \mathrm{d} x^{\beta}$, where $\mathcal{O}\left(h^{2}\right)$ collects all non linear terms in $h$, the coordinates are $x^{0}=c t, x^{1}=x, x^{2}=y, x^{3}=z$, the origin being fixed at the barycenter of the Solar System, and $\eta_{\alpha \beta}$ is the Minkowskian metric. Then, only first order terms in the metric perturbation $h$ are retained. These terms already include all of the possible $(v / c)^{n}$-order expansions of post-Newtonian approach ( $v$ is the velocity of the source and $c$ the velocity of light), but just those up to $(v / c)^{3}$ are needed to reach the micro-arcsecond accuracy required for Gaia.

The global and the local BCRS. In RAMOD a Barycentric Celestial Reference System (BCRS) is identified requiring that a smooth family of space-like hypersurfaces exists with equation $t(x, y, z)=$ constant (see de Felice et al., 2004). The function $t$ can be taken as a time coordinate. On each of these $t(x, y, z)=$ constant hypersurfaces one can choose a set of Cartesian-like coordinates centered at the barycenter of the Solar System (B) and running smoothly as parameters along space-like curves which point to distant cosmic sources. The parameters $x, y, z$, together with the time coordinate $t$, provides a basic coordinate representation of the space-time according to the IAU resolutions. Any tensorial quantity will be expressed in terms of coordinate components relative to coordinate bases induced by the BCRS.

Moreover, the RAMOD geometrical construct allows the existence, at any space-time point, of a unitary four-vector $\mathbf{u}$ which is tangent to the world line of a physical observer at rest with respect to the spatial grid of the BCRS defined as:

$$
u^{\alpha}=\left(-g_{00}\right)^{-1 / 2} \delta_{0}^{\alpha}=\left(1+\frac{h_{00}}{2}\right) \delta_{0}^{\alpha}+\mathcal{O}\left(h^{2}\right) .
$$

The totality of these four-vectors over the space-time forms a vector field which is proportional to a time-like and asymptotically Killing vector field (de Felice et al., 2004). To the order of accuracy required for Gaia, the rest space of $\mathbf{u}$ can be locally identified by a spatial triad of unitary and orthogonal vectors lying on a surface which differs from the $t=$ constant one, but chosen in such a way that their spatial components point to the local coordinate directions as chosen by the BCRS. This frame will be called local BCRS, represented by a tetrad whose spatial axes (the triad) coincide with the local coordinate 
axes, but whose origin is the barycenter of the satellite. This triad is (Bini et al., 2003)

$$
\lambda_{\hat{a}}^{\alpha}=h_{0 a} \delta_{0}^{\alpha}+\left(1-\frac{h_{00}}{2}\right) \delta_{a}^{\alpha}+\mathcal{O}\left(h^{2}\right)
$$

for $a=1,2,3$. In RAMOD any physical measurement refers to the local BCRS.

The proper reference frame for the satellite. The tensorial quantity which expresses a proper reference frame of a given observer is a tetrad adapted to that observer, namely a set of four unitary mutually orthogonal four-vectors $\left\{\lambda_{\hat{\alpha}}\right\}$ one of which, i.e. $\lambda_{\hat{0}}$, is the observer's four-velocity while the other $\lambda_{\hat{a}} \mathrm{~s}$ form a spatial triad of space-like four-vectors. The physical measurements made by the observer (satellite) represented by such a tetrad are obtained by projecting the appropriate tensorial quantities on the tetrad axes. As far as RAMOD is concerned, in the case of a Gaia-like mission, an explicit analytic expression for a tetrad adapted to the satellite four-velocity exists and can be found in (Bini et al., 2003). The spatial axes of this tetrad, $E_{\hat{a}}$, are used to model the attitude of the satellite.

\section{RAMOD and GREM first comparison}

Any comparison between RAMOD and GREM requires that both use the same metric. In the Gaia context the metric perturbation terms can be chosen as $h_{00}=2 w / c^{2}$, $h_{0 i}=w^{i} / c^{3}$, where $w$ and $w^{i}$ are, respectively, the gravitational potential and the vector potential generated by all the sources inside the Solar System according the IAU resolution B1.3 (Soffel et al., 2003).

GREM reproduces in a relativistic framework the classical approach of astrometry, where the quantities which ultimately enter the catalogue are referred to a global inertial reference system, taking into account, one by one and independently from each other, effects such as aberration and parallax. The BCRS is, for this model, the equivalent of the inertial reference system of the classical approach, while the final expression of the star direction in the BCRS is obtained after converting the observed direction into coordinate ones in several steps which divide the effects of the aberration, the gravitational deflection, the parallax, and proper motion (Klioner, 2003). In GREM the observed direction to the source $(\mathbf{s})$ with respect to the local inertial frame $\left(\mathcal{X}^{\alpha}\right)$ of the observer is

$$
s^{i}=-\frac{\mathrm{d} \mathcal{X}^{i}}{\mathrm{~d} \mathcal{X}^{0}} .
$$

The coordinate direction to the light source at the satellite location $\mathbf{x}_{\mathrm{S}}$ is defined by the four-vector $p^{\alpha}=\left(1, p^{i}\right)$, where $p^{i}=c^{-1} d x^{i} / d t, x^{i}$ and $t$ being the BCRS coordinates.

The observables in RAMOD, instead, are the three direction cosines which identify the local line-of-sight to the observed object, relative to a spatial triad $E_{\hat{a}}$ associated to a given observer $\mathbf{u}^{\prime}$; namely:

$$
\cos \psi_{\hat{a}}=\frac{P\left(u^{\prime}\right)_{\alpha \beta} k^{\alpha} E_{\hat{a}}^{\beta}}{\left(P\left(u^{\prime}\right)_{\alpha \beta} k^{\alpha} k^{\beta}\right)^{1 / 2}} \equiv \mathbf{e}_{\hat{a}},
$$

where the final $\mathbf{e}_{\hat{a}}$ has to be intended a shorthand notation for $\cos \psi_{\hat{a}}, k^{\alpha}$ is the four-vector tangent to the null geodesic connecting the star to the observer, and $P\left(u^{\prime}\right)$ is the operator which projects on the rest-space of $\mathbf{u}^{\prime}$; all the quantities are obviously computed at the event of the observation. The RAMOD formulation naturally entangles in the previous formula every GR "effect" when (as in Bini et al., 2003) the attitude frame $E_{\hat{a}}$ is that of a Gaia-like observer. Therefore, to retrieve the aberration in RAMOD, one needs to specialize Eq. 3.2 to the case of a tetrad $\left\{\tilde{\lambda}_{\hat{\alpha}}\right\}$ adapted to the center of mass of the satellite assumed with no attitude parameters. In this case the observation equation will give a 
relation between the "aberrated" direction represented by the cosines as measured by the satellite and the "aberration-free" direction. The latter is given by quantity $\bar{l}^{\alpha}=P_{\beta}^{\alpha}(u) k^{\beta}$ referred to the local BCRS frame $\left\{\lambda_{\hat{a}}\right\}$, where $\bar{l}^{\alpha}$ was introduced in RAMOD (de Felice et al., 2004) as the unitary four-vector which represents the local line-of-sight of the photon as seen by u. The tetrad $\left\{\tilde{\lambda}_{\hat{a}}\right\}$ differ from the local BCRS's $\left\{\lambda_{\hat{a}}\right\}$ for a boost transformation with four-velocity $u_{s}^{\alpha}$ of the satellite (Jantzen et al., 1992, Bini et al., 2003) and plays the same role of the CoMRS (Center-of-Mass Reference System) defined for Gaia (Klioner, 2004). Considering the IAU metric, we obtain ( $v^{i}$ stands for the spatial coordinate velocity of the satellite)

$$
\begin{aligned}
\tilde{\mathbf{e}}_{\hat{a}} \approx & \bar{l}^{a}+\frac{1}{c}\left[-v^{a}+\left(\delta_{i j} v^{i} \bar{l}{ }^{j}\right) \bar{l}^{a}\right]+\frac{1}{c^{2}}\left\{w \bar{l}^{a}-\frac{1}{2}\left(\delta_{i j} v^{i} \bar{l}^{j}\right) v^{a}+\left[\left(\delta_{i j} v^{i} \bar{l}^{j}\right)^{2}-\frac{1}{2} v^{2}\right] \bar{l}^{a}\right\} \\
& +\frac{1}{c^{3}}\left\{-2 w v^{a}-\frac{1}{2}\left(\delta_{i j} v^{i} \bar{l}^{j}\right)^{2} v^{a}+\bar{l}^{a}\left[3 w\left(\delta_{i j} v^{i} \bar{l}^{j}\right)\right.\right. \\
& \left.\left.+\left(\delta_{i j} v^{i} \bar{l}^{j}\right)^{3}-\frac{1}{2} v^{2}\left(\delta_{i j} v^{i} \bar{l}^{j}\right)+w\left(\delta_{i j} v^{i} \bar{l}^{j}\right)\right]\right\}+\mathcal{O}\left(v^{4} / c^{4}\right)
\end{aligned}
$$

where $\tilde{\mathbf{e}}_{\hat{a}}$ are the cosines related to the tetrad without the attitude parameters.

At a first glance, the last expression shows differences in terms up to the $(v / c)^{2}$ order (note in particular the term $w \bar{l}^{a}$ ) and of the $(v / c)^{3}$ order which cannot allow to straightforwardly compare, as expected, the above expression to the GREM vectorial one (see Klioner, 2003), where the aberration is expressed in terms of a vector $\mathbf{n}=p^{i} / p$, which represents the "aberration-free" coordinate line of sight of the observed star at the position of the satellite momentarily at rest. To compare formula 3.3 with GREM's formula, $\mathbf{n}$ and $\bar{l}^{\alpha}$ we need to reduce $\bar{l}^{\alpha}$ to its coordinate Euclidean expression. In RAMOD, as said, $\bar{l}^{\alpha}$ represents the normalized local line-of-sight of the observed star as seen by the local barycentric observer $\mathbf{u}$. From the physical point of view $\mathbf{n}$ and $\bar{l}^{\alpha}$ have the same meaning, as the observed "aberration free" direction to the star. From the definition of $n^{i}$, we recover $n^{i}=p^{i}\left(1+h_{00}+h_{0 i} p^{i}\right)+\mathcal{O}\left(h^{2}\right)$. On the other hand, using the definition of $\bar{l}^{\alpha}$ in de Felice et al., 2004, and from $u^{0}=\left(-g_{00}\right)^{-1 / 2}$ and $k^{i} / k^{0}=c^{-1} \mathrm{~d} x^{i} / \mathrm{d} t \equiv p^{i}$, it can be easily shown that its spatial components can be approximated as

$$
\bar{l}^{i}=n^{i}\left(1-\frac{h_{00}}{2}\right)+\mathcal{O}\left(\frac{v^{4}}{c^{4}}\right) .
$$

Combining Eq. 3.3 with 3.4 and setting $\left(\delta_{i j} v^{i} n^{j}\right) \equiv \mathbf{v} \cdot \mathbf{n}$ to ease the notation, we obtained

$$
\begin{aligned}
\tilde{\mathbf{e}}_{\hat{a}}= & n^{a}+\frac{1}{c}\left[-v^{a}+(\mathbf{v} \cdot \mathbf{n}) n^{a}\right]+\frac{1}{c^{2}}\left\{-\frac{1}{2}(\mathbf{v} \cdot \mathbf{n}) v^{a}+\left[(\mathbf{v} \cdot \mathbf{n})^{2}-\frac{1}{2} v^{2}\right] n^{a}\right\}+ \\
& \frac{1}{c^{3}}\left\{-2 w v^{a}-\frac{1}{2}(\mathbf{v} \cdot \mathbf{n})^{2} v^{a}+(\mathbf{v} \cdot \mathbf{n}) n^{a}\left[2 w+(\mathbf{v} \cdot \mathbf{n})^{2}-\frac{1}{2} v^{2}\right]\right\}+\mathcal{O}\left(\frac{v^{4}}{c^{4}}\right) .
\end{aligned}
$$

In this way the right-hand side of the aberration expression of RAMOD is rewritten with the GREM quantities at the $(v / c)^{3}$ order. The final step is to find a relation between $\tilde{\mathbf{e}}_{\hat{a}}$ and $s^{i}$. Using the definition of the projection operator and the tetrad property $\left(\lambda_{\alpha}^{\hat{\mu}} \lambda_{\hat{\mu} \beta}=g_{\alpha \beta}\right)$, we have:

$$
\tilde{\mathbf{e}}_{\hat{a}} \equiv \frac{P(u)_{\alpha \beta} k^{\alpha} \tilde{\lambda}_{\hat{a}}^{\beta}}{\left(P(u)_{\alpha \beta} k^{\alpha} k^{\beta}\right)^{1 / 2}}=\frac{k^{\alpha} \tilde{\lambda}_{\alpha}^{\hat{a}}}{\left|g_{\alpha \beta} u^{\alpha} k^{\beta}\right|}=-\frac{k^{\alpha} \tilde{\lambda}_{\alpha}^{\hat{a}}}{g_{\alpha \beta} \tilde{\lambda}_{\hat{0}}^{\alpha} k^{\beta}}=\frac{k^{\alpha} \tilde{\lambda}_{\alpha}^{\hat{a}}}{k^{\beta} \tilde{\lambda}_{\beta}^{\hat{0}}}=\frac{\mathrm{d} \tilde{x}^{\hat{a}}}{\mathrm{~d} \tilde{x}^{\hat{0}}} .
$$

The tetrad components of the light ray can be directly associated to CoMRS coordinates, i.e. to a coordinate-induced tetrad (as done in Klioner, 2004), if the boosted local BCRS 
tetrad coordinates $\tilde{x}^{\hat{\alpha}}$ are equivalent to the CoMRS ones $\mathcal{X}^{\alpha}$. This is true if the origins of the two reference systems coincide and only locally, since the tetrad are not in general holonomic. So, in the case of Gaia, from 3.1 it is $\tilde{\mathbf{e}}_{\hat{a}}=-s^{a}$. Therefore one can finally write relation 3.5 (where $\mathbf{n} \cdot \mathbf{n}=1$ and $v^{2}=\delta_{i j} v^{i} v^{j} \equiv \mathbf{v} \cdot \mathbf{v}$ )

$$
\begin{aligned}
s^{a}= & -n^{a}+\frac{1}{c}[\mathbf{n} \times(\mathbf{v} \times \mathbf{n})]^{a}+\frac{1}{c^{2}}\left\{(\mathbf{v} \cdot \mathbf{n})[\mathbf{n} \times(\mathbf{v} \times \mathbf{n})]^{a}+\frac{1}{2}[\mathbf{v} \times(\mathbf{n} \times \mathbf{v})]^{a}\right\} \\
& +\frac{1}{c^{3}}\left\{\left[(\mathbf{v} \cdot \mathbf{n})^{2}+2 w\right][\mathbf{n} \times(\mathbf{v} \times \mathbf{n})]^{a}+\frac{1}{2}(\mathbf{v} \cdot \mathbf{n})[\mathbf{v} \times(\mathbf{n} \times \mathbf{v})]^{a}\right\}+\mathcal{O}\left(\frac{v^{4}}{c^{4}}\right)
\end{aligned}
$$

which is the formula for the aberration in GREM.

\section{Conclusions}

The direction cosines being physical quantities not depending on the coordinates, are a powerful tool to compare the astrometric relativistic models: their physical meaning allow us to correctly interpret the astrometric parameters in terms of coordinate quantities. This justified the conversion of the physical stellar proper direction of RAMOD into its analogous Euclidean coordinate counterpart, which ultimately leads to the derivation of a GREM-style aberration formula 3.7. Note, also, that the observables of RAMOD can be matched with components of the observed $s^{i}$ of GREM only if the origins of the boosted local BCRS tetrad in RAMOD and of the CoMRS in GREM coincide.

Moreover, the direction cosines (i.e. the astrometric measurements strictly dependent on the mathematical characterization of the attitude) taken as a function of the local lineof-sight (the physical one), at the time of observation, allow to fix the boundary conditions needed to solve the master equations and to determine uniquely the star coordinates. The vector $\mathbf{n}$, i.e. the "aberration-free" counterpart of $l^{\alpha}$ in GREM, is instead used to derive the aberration effect (in a coordinate language) and there is no need to connect it with a RAMOD-like boundary value problem.

\section{Acknowledgements}

This presentation was supported by the IAU grants.

\section{References}

Soffel M., Klioner S. A., Petit G., Wolf P., Kopeikin S. M., Bretagnon P., Brumberg V. A., Capitaine N., Damour T., Fukushima T. et al., 2003, Astron. J., 126, 2687

Eds. Turon C., O'Flaherty K. S., \& Perryman M. A. C., 2005, The Three-Dimensional Universe with Gaia, Publ. Astron. Soc. Pac., 120, 38

Bini D., Crosta M., \& de Felice F. 2003, Class. Quantum Grav., 20, 4695

de Felice F., crosta M., Vecchiato A., Lattanzi M. G., \& Bucciarelli B., 2004, Astrophys. J., 607, 580.

de Felice F., Vecchiato A., Crosta M., Lattanzi M. G., \& Bucciarelli B., 2006 Astrophys. J., 653, 1552

de Felice F \& Preti G., 2006, Class. Quantum Grav., 23, 5467

Klioner S. A., 2003 Astron. J., 125, 1580

Gravitation, Misner C. W., Thorne, K. S., \& Wheeler J. A., 1973 San Francisco: W.H. Freeman and $\mathrm{Co}$.

Jantzen R. T., Carini P., Bini D., 1992 Ann. Phys., 215, 1

Klioner S. A., 2004 Phys. Rev. D, 69, 124001 